\title{
Double degeneracy associated with hidden symmetries in the asymmetric two-photon Rabi model
}

\author{
You-Fei Xie ${ }^{1}$ and Qing-Hu Chen $\oplus^{1,2, *}$ \\ ${ }^{1}$ Zhejiang Province Key Laboratory of Quantum Technology and Device, Department of Physics, \\ Zhejiang University, Hangzhou 310027, China \\ ${ }^{2}$ Collaborative Innovation Center of Advanced Microstructures, Nanjing University, Nanjing 210093, China
}

(Received 22 February 2021; accepted 28 June 2021; published 16 July 2021)

\begin{abstract}
In this paper, we uncover the elusive level crossings in a subspace of the asymmetric two-photon quantum Rabi model (tpQRM) when the bias parameter of qubit is an even multiple of the renormalized cavity frequency. Due to the absence of any explicit symmetry in the subspace, this double degeneracy at the crossing points implies the existence of the hidden symmetry. It is found that the number of the doubly degenerate crossing points in the asymmetric tpQRM is comparable to that in asymmetric one-photon QRM in terms of the same order of the constrained conditions. The bias parameter required for the occurrence of level crossings in the asymmetric tpQRM is characteristically different from that at a multiple of the cavity frequency in the asymmetric one-photon QRM, suggesting the different hidden symmetries in the two asymmetric QRMs.
\end{abstract}

DOI: 10.1103/PhysRevResearch.3.033057

\section{INTRODUCTION}

The simplest interaction between a two-level system (qubit) and a single-mode bosonic cavity (oscillator) was described by the quantum Rabi model (QRM) [1,2], which is, thus, a fundamental textbook model in quantum optics [3]. It has been demonstrated in many advanced solid devices, such as circuit quantum electrodynamics (QED) system [4,5], trapped ions [6], and quantum dots [7] from weak coupling to the ultrastrong coupling, even deep strong coupling between the artificial atom and resonators [8-10].

In contrast to the conventional cavity QED system, the artificial qubit appears in modern solid devices usually contains both the splitting $\Delta$ and the bias $\epsilon$ between the two-qubit states, thus, the so-called asymmetric QRM is ubiquitous. Driven by the proposals and experimental realizations of the various QRMs, the asymmetric two-photon QRM (tpQRM) are also realized or stimulated to explore new quantum effects [11-13]. The typical two asymmetric QRMs can be generally written in a unified way as

$$
H_{p}^{\epsilon}=-\frac{\Delta}{2} \sigma_{x}+\frac{\epsilon}{2} \sigma_{z}+\omega a^{\dagger} a+g\left[\left(a^{\dagger}\right)^{p}+a^{p}\right] \sigma_{z},
$$

where the first two terms fully describe a qubit with the energy splitting $\Delta$ and the biases $\epsilon, \sigma_{x, z}$ are the Pauli matrices, $a^{\dagger}$ and $a$ are the creation and annihilation operators with the cavity frequency $\omega$, and $g$ is the qubit-cavity coupling strength. $p=1,2$ denote the one-photon and two-photon QRMs, respectively. In the superconducting flux qubit [8,9], $\Delta$ is the tunnel coupling between the two persistent current states $\epsilon=$

\footnotetext{
*qhchen@zju.edu.cn

Published by the American Physical Society under the terms of the Creative Commons Attribution 4.0 International license. Further distribution of this work must maintain attribution to the author(s) and the published article's title, journal citation, and DOI.
}

$2 I_{p}\left(\Phi-\Phi_{0} / 2\right)$ with $I_{p}$ as the persistent current in the qubit loop, $\Phi$ as the an externally applied magnetic flux, and $\Phi_{0}$ as the flux quantum. The flux qubit is usually manipulated by the external magnetic flux and the persistent currents.

For the symmetric case $(\epsilon=0)$, the one-photon QRM possesses $\mathbb{Z}_{2}$-symmetry (parity), i.e., $\left[H_{1}^{0}, \hat{P}_{1}\right]=0$ with the parity operator $\hat{P}_{1}=-\sigma_{x} \exp \left(i \pi a^{\dagger} a\right)$, whose eigenvalues are \pm 1 , whereas the tpQRM has $\mathbb{Z}_{4}$ symmetry, i.e., $\left[H_{2}^{0}, \hat{P}_{2}\right]=0$ with the parity operator $\hat{P}_{2}=-\sigma_{x} \exp \left(i \pi a^{\dagger} a / 2\right)$, whose eigenvalues are the quartic roots of unity $\pm 1, \pm i[14,15]$. Hence, the whole Hilbert space separates into two and four infinitedimensional subspaces in one-photon QRM and the tpQRM, respectively.

An analytical exact solution of the one-photon QRM has been found by Braak in the Bargmann space representation [16]. It was quickly reproduced in the more familiar Hilbert space using the Bogoliubov operator approach (BOA) by Chen et al. [17]. Moreover, the BOA can be easily extended to the tpQRM, and solutions in terms of a $G$-function, which shares the common pole structure with Braak's $G$ function for the one-photon QRM, are also found. It was soon realized that the $G$ function can be constructed in terms of the mathematically well-defined Heun confluent function [18]. These studies have stimulated extensive interests in various QRMs [19-27]. For more theoretical details in this field, one may refer to recent review articles [28-30].

Level crossing is very helpful to identify the symmetry in quantum systems. The quasiexact energies at the crossing points in the symmetric QRMs, also called Juddian solutions [31], have been found $20 \mathrm{yr}$ ago [32]. The Juddian solutions are corresponding to the doubly degenerate states and can be constructed with the terminated polynomials. These quasiexact energies now can also be easily derived with the help of the pole structure of the $G$ function in both the one-photon [16] and the two-photon [17] QRMs.

The presence of the qubit bias term $\frac{\epsilon}{2} \sigma_{z}$ breaks $\mathbb{Z}_{2}$-parity symmetry of the QRM, so no any obvious symmetry remains 
in the asymmetric QRM [18,24,33]. So the level crossings generally do not appear [16]. Surprisingly, when $\epsilon$ is a multiple of the cavity frequency $\omega$, it is observed in Ref. [18] that the energy-level crossings reappear. This double degeneracy at the level crossing point in the asymmetric model indeed reflects an unknown hidden symmetry. It must be different from the parity symmetry because the parity symmetry is broken by any biases. Very recently, the hidden $\mathbb{Z}_{2}$ symmetry different from the parity symmetry in the asymmetric QRM is discussed based on the numerical calculation on the energy eigenstates [34] and symmetry operators [35,36] for small integers $\epsilon / \omega$.

While in the tpQRM, the qubit bias term $\frac{\epsilon}{2} \sigma_{z}$ reduces the original $\mathbb{Z}_{4}$ symmetry to the $\mathbb{Z}_{2}$ symmetry. In the asymmetric tpQRM, the $\mathbb{Z}_{2}$ symmetry corresponding to the parity operator $\hat{P}_{2 p}=\exp \left(i \pi a^{\dagger} a\right)$ only acts in the bosonic Hilbert spaces, the whole Hilbert space then only divides into two invariant subspaces: even and odd number Fock states, which can be still labeled by the Bargmann index $q=1 / 4$ and 3/4 [14] . For the symmetric tpQRM, the standard Juddian solutions are level crossings within the same $q$ subspace. The second type of level crossings of the eigenstates in different $q$ subspaces [32] was also found recently [37,38]. In the asymmetric tpQRM since $\mathbb{Z}_{4}$ symmetry reduces to $\mathbb{Z}_{2}$ symmetry, the level crossings within the same $q$ subspace would generally disappear, whereas the second type of the level crossings in the different $q$ subspaces remains robust due to the remaining $\mathbb{Z}_{2}$ symmetry. Contrary to the one-photon asymmetric QRM [33], the level crossing within the same $q$ subspace in the asymmetric tpQRM is elusive and is observed here. In this paper, we will uncover such a kind of level crossings irrelevant to any explicit symmetry.

The paper is structured as follows: In Sec. II, we briefly review the solutions to the asymmetric one-photon QRM in the framework of the BOA approach and corroborate the previous observed doubly degenerate states in the BOA frame. We extend the BOA to study the asymmetric tpQRM and derive the analytical exact solutions in Sec. III. In Sec. IV, we demonstrate the level crossings within the same $q$ subspace of the asymmetric tpQRM. The characteristics of level crossings in the two asymmetric QRMs are discussed in Sec. V. The last section contains some concluding remarks. Appendix A confirms the conjecture in both the asymmetric one-photon $\mathrm{QRM}$ and the tpQRM that the two vanishing coefficients yield the same solutions, thus, leading to the true level crossings. Appendix B presents the nondegenerate exceptional solutions for both the asymmetric one-photon QRM and the tpQRM.

\section{ASYMMETRIC QUANTUM RABI MODEL IN THE BOA}

For the asymmetric one-photon QRM, when the bias parameter $\epsilon$ is a multiple of the cavity frequency, the level crossings appear again in the spectra even without any explicit known symmetry in the system [18,24]. It should be noted that here $\epsilon$ in accord with the standard qubit Hamiltonian $[8,9,34,39]$ is twice of that used in Refs. [16,18,24].

In this section, we revisit the asymmetric one-photon QRM by the BOA. We first briefly review the solutions in the BOA framework [17], then we can describe the level crossings in the BOA alternatively, which is essentially equivalent to the Bargmann space approach. Moreover, this scheme can be easily extended to the asymmetric tpQRM in the next sections.

\section{A. Solutions in the BOA}

By two Bogoliubov transformations,

$$
A=a+g / \omega, \quad B=a-g / \omega,
$$

the wave function can be expressed as the series expansions in terms of the $A$ operator,

$$
|A\rangle=\left(\begin{array}{c}
\sum_{n=0}^{\infty} \sqrt{n !} e_{n}|n\rangle_{A} \\
\sum_{n=0}^{\infty} \sqrt{n !} f_{n}|n\rangle_{A}
\end{array}\right)
$$

where $e_{n}$ and $f_{n}$ are the expansion coefficients and in terms of the $B$ operator,

$$
|B\rangle=\left(\begin{array}{c}
\sum_{n=0}^{\infty}(-1)^{n} \sqrt{n !} c_{n}|n\rangle_{B} \\
\sum_{n=0}^{\infty}(-1)^{n} \sqrt{n !} d_{n}|n\rangle_{B}
\end{array}\right),
$$

with two coefficients $c_{n}$ and $d_{n},|n\rangle_{A}$ and $|n\rangle_{B}$ are called extended coherent states [40].

By the Schrödinger equation, we get the linear relation for two coefficients $e_{m}$ and $f_{m}$ with the same index $m$ as [17]

$$
e_{m}=\frac{\Delta}{2\left(m \omega-g^{2} / \omega+\frac{\epsilon}{2}-E\right)} f_{m},
$$

and the coefficient $f_{m}$ can be defined recursively,

$$
\begin{aligned}
(m+1) f_{m+1}= & \frac{1}{2 g}\left(m \omega+3 g^{2} / \omega-\frac{\epsilon}{2}-E\right. \\
& \left.-\frac{\Delta^{2}}{4\left(m \omega-g^{2} / \omega+\frac{\epsilon}{2}-E\right)}\right) f_{m}-f_{m-1},
\end{aligned}
$$

with $f_{0}=1$. Similarly, the two coefficients $c_{m}$ and $d_{m}$ satisfy

$$
d_{m}=\frac{\Delta}{2\left(m \omega-g^{2} / \omega-\frac{\epsilon}{2}-E\right)} c_{m},
$$

and the recursive relation is given by

$$
\begin{aligned}
(m+1) c_{m+1}= & \frac{1}{2 g}\left(m \omega+3 g^{2} / \omega+\frac{\epsilon}{2}-E\right. \\
& \left.-\frac{\Delta^{2}}{4\left(m \omega-g^{2} / \omega-\frac{\epsilon}{2}-E\right)}\right) c_{m}-c_{m-1},
\end{aligned}
$$

with $c_{0}=1$.

If both wave-functions (3) and (4) are the true eigenfunction for a nondegenerate eigenstate with eigenvalue $E$, they should be, in principle, only different by a complex constant $z$, i.e., $|A\rangle=z|B\rangle$. Projecting both sides onto the original vacuum state $|0\rangle$, using $\sqrt{n !}\langle 0 \mid n\rangle_{A}=(-1)^{n} \sqrt{n !}\langle 0 \mid n\rangle_{B}=$ $e^{-(g / \omega)^{2} / 2}(g / \omega)^{n}$ and eliminating the ratio constant $z$ gives

$$
\sum_{n=0}^{\infty} e_{n}\left(\frac{g}{\omega}\right)^{n} \sum_{n=0}^{\infty} d_{n}\left(\frac{g}{\omega}\right)^{n}=\sum_{n=0}^{\infty} f_{n}\left(\frac{g}{\omega}\right)^{n} \sum_{n=0}^{\infty} c_{n}\left(\frac{g}{\omega}\right)^{n},
$$


with the help of Eqs. (5) and (7), one arrives at the one-photon $G$ function,

$$
\begin{aligned}
G_{1 p}= & \left(\frac{\Delta}{2}\right)^{2}\left[\sum_{n=0}^{\infty} \frac{f_{n}}{n \omega-g^{2} / \omega+\frac{\epsilon}{2}-E}\left(\frac{g}{\omega}\right)^{n}\right] \\
& \times\left[\sum_{n=0}^{\infty} \frac{c_{n}}{n \omega-g^{2} / \omega-\frac{\epsilon}{2}-E}\left(\frac{g}{\omega}\right)^{n}\right] \\
& -\sum_{n=0}^{\infty} f_{n}\left(\frac{g}{\omega}\right)^{n} \sum_{n=0}^{\infty} c_{n}\left(\frac{g}{\omega}\right)^{n} .
\end{aligned}
$$

This $G$ function was first derived by Braak [16] using Bargmann space approach, and later reproduced by Chen et al. [17]. In the series expansions of Eq. (10), the coefficients before $(g / \omega)^{n}$ depend on $g$ also, the $G$ function actually does not diverge for arbitrary large $g$ except at the pole energies. The discussion of the convergence of the $G$ function is given in the Supplemental Material of Ref. [16]. We then discuss the level crossing of the asymmetric QRM in terms of the BOA framework described above.

\section{B. Doubly degenerate states}

The two types of pole energies appear in the one-photon $G$-function (10) as

$$
\begin{aligned}
E_{N}^{A} & =N \omega-g^{2} / \omega+\frac{\epsilon}{2}, \quad N=0,1,2, \ldots, \\
E_{M}^{B} & =M \omega-g^{2} / \omega-\frac{\epsilon}{2}, \quad M=0,1,2, \ldots
\end{aligned}
$$

They are labeled with the type- $A$ and type- $B$ pole energy, respectively. If

$$
\epsilon=(M-N) \omega,
$$

these two pole energies are the same,

$$
E_{N}^{A}(g)=E_{M}^{B}(g)=\frac{1}{2}(M+N) \omega-g^{2} / \omega .
$$

Note that $\epsilon$ should be a multiple of the cavity frequency $\omega$ under the condition (13). In this paper, we only consider $M>N$ so that $\epsilon$ is positive. For the case of $M<N$, the extension is achieved straightforwardly by changing $\epsilon$ into $-\epsilon$ and interchanging $M$ and $N$.

From Eqs. (5) and (7), one immediately notes that the coefficient $e_{N}\left(d_{M}\right)$ would diverge at the same pole energy (14). It does not make sense if some coefficients in the series expansion of a wave function really become infinity. A normalizable wave function should consist of the global property, i.e., the finite inner product, so the series expansion coefficients in the wave-functions (3) and (4) should be analytic and vanish as or before $n \rightarrow \infty$.

To achieve a physics state, at the pole energy (14), the numerator of the right-hand side of Eqs. (5) and (7) should also vanish so that $e_{N}\left(d_{M}\right)$ remains finite, which results in

$$
f_{N}(M, g)=0 ; \quad c_{M}(N, g)=0 .
$$

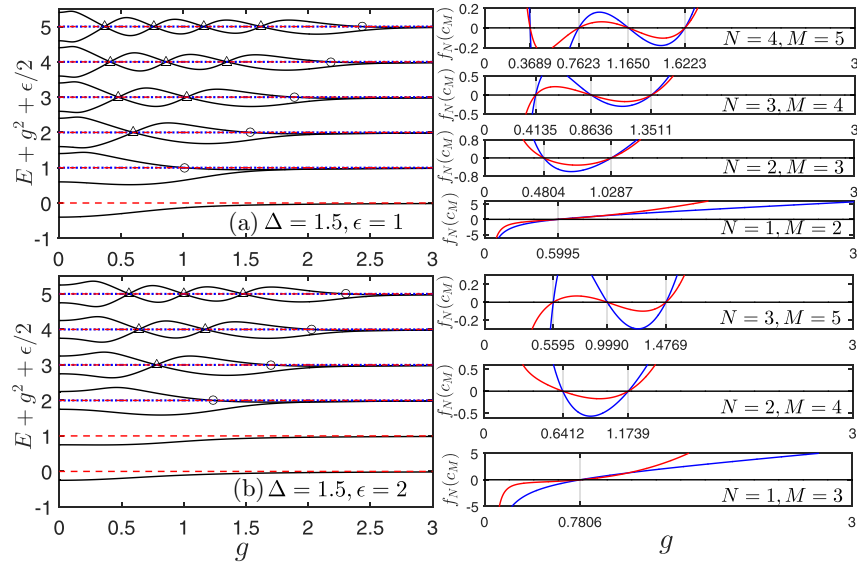

FIG. 1. Energy spectra $E+g^{2}+\epsilon / 2$ for $\omega=1, \Delta=1.5, \epsilon=$ 1 (a) and $\epsilon=2$ (b) in left panels. The horizontal blue dotted lines correspond to the pole energy ones for $E_{N}^{A}$ and the red dashed lines to $E_{M}^{B}$. Only the overlapped pole lines with $N>0$ allow for the true level crossings. The triangles denotes the doubly degenerate crossing points. Circles indicate the nondegenerate exceptional solutions by Eq. (B3). $f_{N}(M, g)$ (blue) and $c_{M}(N, g)$ (red) curves are shown in the right panels. The zeros are exactly corresponding to the triangles in the left spectra.

Note that $f_{N}$ and $c_{M}$ can be obtained using the following threeterm recurrence relations from (6) and (8) with energy (14), respectively,

$$
\begin{aligned}
(n+1) f_{n+1}= & \frac{1}{2 g}\left[4 g^{2} / \omega+(n-M) \omega-\frac{\Delta^{2}}{4 \omega(n-N)}\right] f_{n} \\
& -f_{n-1}, \\
(n+1) c_{n+1}= & \frac{1}{2 g}\left[4 g^{2} / \omega+(n-N) \omega-\frac{\Delta^{2}}{4 \omega(n-M)}\right] c_{n} \\
& -c_{n-1} .
\end{aligned}
$$

If $\Delta, M, N$ are given, two equations in Eq. (15) would provide the coupling strength $g$ in the energy spectra where the energy levels intersect with the same pole line described by Eq. (14).

A mathematical proof to the conjecture that $f_{N}(M, g)=0$ and $c_{M}(N, g)=0$ could give the same real and positive solutions for the coupling strength $g$ was given in Ref. [33]. Li and Batchelor [24] have analyzed the relation between the number of the exceptional points and the model parameters $(\Delta$ and $\epsilon$ ) and numerically found that the number of positive roots from these two equations are the same for integer $\epsilon / \omega$. But we confine us here to a closed-form proof for small values of $N$ and $M$ and numerical confirmation for large $N$ and $M$. In Appendix A 1, we show the same crossing points by two equations in Eq. (15) for the fixed integers $N$ and $M$.

The true level crossings in the asymmetric one-photon QRM for integer $\epsilon / \omega$ can be exhibited in the energy spectra. To visualize this phenomena, we, respectively, present the energy spectra $E+g^{2} / \omega+\frac{\epsilon}{2}$ for $\Delta=1.5$ and $\Delta=3$ in the left panels of Figs. 1 and 2 with $\epsilon=1,2, \omega=1$. The dotted and dashed horizontal lines denote different types of pole lines. Obviously, if the two types of pole lines cannot coincide, the level crossings cannot happen. $f_{N}(M, g)$ and $c_{M}(N, g)$ curves are plotted in the right panels. By Eq. (A1), one finds 

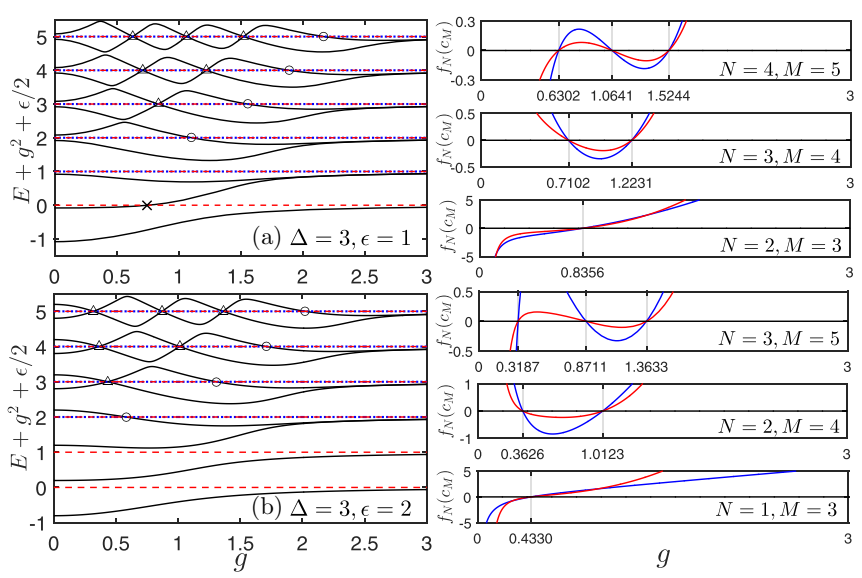

FIG. 2. Notations are the same as those in Fig. 1 except for $\Delta=$ 3 . Note that the doubly degenerate crossing point is absent in the $N=1, \quad M=2$ overlapped line in (a) due to $\Delta>2 \sqrt{2}$ here.

$g=0.5995$ for $N=1, M=2$ at $\Delta=1.5$, consistent with the spectra in Fig. 1(a). For $\Delta=3$, see Eq. (A2), no real positive solution can be found in this case, so level crossings cannot occur in the overlapped line with $N=1$ in Fig. 2(a).

At $\Delta=1.5$, we can obtain two solutions for $g$ as 0.4804 , 1.0287 for $N=2$ and $M=3$ by Eq. (A4), agreeing well with two crossing points in the pole line with $N=2$ and $M=3$ shown in Fig. 1(a). For $\Delta=3$, we only find one real positive $g=0.8356$, consistent with the spectra in Fig. 2(a).

Associated with the overlapped $N=1$ and $M=3$ pole lines, one can obtain $g=0.7806$ for $\Delta=1.5$, and $g=0.4330$ for $\Delta=3$ by Eq. (A6), consistent with the crossing points in the calculated spectrum in Figs. 1(a) and 2(a).

For large values of $M$ and $N$ as shown in the right panels of Figs. 1 and 2, both $f_{N}(M, g)$ and $c_{M}(N, g)$ curves provide the same zeros for all cases.

Now we will further demonstrate explicitly that any crossing point found above is corresponding to a doubly degenerate state in the BOA framework. At the crossing point, looking at (5) since both the numerator $f_{N}(g)$ and the denominator vanish, $e_{N}$ would be arbitrary. If we set

$$
e_{N}=-\frac{4 g}{\Delta} f_{N-1}
$$

from Eq. (6) we know $f_{N+1}=0$, further $e_{N+1}=0$, and all coefficients $f_{k}$ and $e_{k}$ for $k>N+1$ vanish. So the infinite series expansion in the wave-function (4) terminates with finite $N$ as

$$
|A\rangle_{N}=\left(\begin{array}{l}
\sum_{n=0}^{N} \sqrt{n !} e_{n}|n\rangle_{A} \\
\sum_{n=0}^{N-1} \sqrt{n !} f_{n}|n\rangle_{A}
\end{array}\right)
$$

Similarly, the infinite series expansion in the wave-function (4) terminates with finite $M$ as

$$
|B\rangle_{M}=\left(\begin{array}{l}
\sum_{n=0}^{M-1}(-1)^{n} \sqrt{n !} c_{n}|n\rangle_{B} \\
\sum_{n=0}^{M}(-1)^{n} \sqrt{n !} d_{n}|n\rangle_{B}
\end{array}\right),
$$

where

$$
d_{M}=-\frac{4 g}{\Delta} c_{M-1}
$$

Interestingly, both wave functions terminate at finite terms. Because these two wave functions are not obtained from the $G$ function based on the proportionality (9), so they are different $|A\rangle_{N} \neq|B\rangle_{M}$, leading to doubly degenerate states. Since the degenerate eigenfunctions $|A\rangle_{N}$ and $|B\rangle_{M}$ are given as finite polynomials in the extended coherent state basis $\left\{|n\rangle_{A}\right\}$ and $\left\{|n\rangle_{B}\right\}$ (see also Ref. [41]), these states are the quasiexact solutions of the asymmetric QRM.

At this stage, we can simply discuss the number of the doubly degenerate crossing points associated with the given $N$ type- $A$ pole line. $f_{N}\left(M>N, x=4 g^{2}\right)$ derived by Eq. (16) is a polynomial with $N$ terms. Its zero would generally give around $N$ roots, indicating that there are around $N$ doubly degenerate crossing points along the $N$ type- $A$ pole line in the energy spectra. For large $\Delta$, the number of the roots could be slightly less than $N$ as shown in Fig. 2 whereas for small $\Delta$, we can actually have just $N$ roots in Fig. 1.

The nondegenerate exceptional points can be generated if only one energy level intersects with the energy pole line alone, which are analyzed in Appendix B 1. In the left panels of Figs. 1 and 2, the nondegenerate exceptional points are indicated by open circles and crosses. All the open circles are given by zeros of the nondegenerate exceptional $G$-function (B3), whereas a cross in Fig. 2(a) is solved by $G$-function $G_{1 p}^{\text {non,2B }}$ associated with the type- $B$ pole lines, cf. Eq. (B2).

Overall, one can find that the previous main results in the asymmetric QRM based on the Bargmann space approach, see Ref. [24] and references therein, can be well described in the BOA framework in a self-contained way. Nevertheless, the level crossings in asymmetric tpQRM have not been observed in the literature, to the best of our knowledge. Note that the $G$ function by the direct application of the Bargmann space approach to the tpQRM [42] has no pole structure and, thus, could not give qualitative insight into the behavior of the spectral collapse [15] and the level crossing. As far as we know, the $G$ function with its pole structure for the tpQRM has only been found using the BOA $[14,17,43]$ and, in particular, has so far not been derived using the Bargmann space method in the literature. Therefore, it is perhaps irreplaceable, at the moment, to employ the BOA to explore the level crossings in the asymmetric tpQRM, which is just the main topic of this paper.

\section{ASYMMETRIC TWO-PHOTON RABI MODEL AND SOLUTIONS USING THE BOA}

To facilitate the BOA approach, we write the asymmetric tpQRM Hamiltonian $H_{2}^{\epsilon}$ in Eq. (1) with the following matrix form:

$H_{2}^{\epsilon}=\left(\begin{array}{cc}\omega a^{\dagger} a+g\left(a^{\dagger 2}+a^{2}\right)+\frac{\epsilon}{2} & -\frac{\Delta}{2} \\ -\frac{\Delta}{2} & \omega a^{\dagger} a-g\left(a^{\dagger 2}+a^{2}\right)-\frac{\epsilon}{2}\end{array}\right)$.

It is connected with $\operatorname{su}(1,1)$ Lie algebra [14],

$$
K_{0}=\frac{1}{2}\left(a^{\dagger} a+\frac{1}{2}\right), \quad K_{+}=\frac{1}{2} a^{\dagger 2}, \quad K_{-}=\frac{1}{2} a^{2},
$$

which obey spinlike commutation relations $\left[K_{0}, K_{ \pm}\right]=$ $\pm K_{ \pm},\left[K_{+}, K_{-}\right]=-2 K_{0}$. 
Then we apply a squeezing operator $S_{1}=e^{(r / 2)\left(a^{2}-a^{\dagger 2}\right)}$ to diagonalize the bosonic part of the above Hamiltonian and the parameter $r$ is to be fixed later. In terms of the $K_{0}, K_{ \pm}$, the transformed Hamiltonian is derived as

$$
H_{2}^{\epsilon}=\left(\begin{array}{cc}
\beta\left(2 K_{0}\right)+\frac{\epsilon-\omega}{2} & -\frac{\Delta}{2} \\
-\frac{\Delta}{2} & H_{22}
\end{array}\right),
$$

where $\beta=\omega \sqrt{1-4\left(\frac{g}{\omega}\right)^{2}}<\omega$ can be termed as the renormalized cavity frequency owing to the fact that it is just a $g$-dependent prefactor of the free photon number operators $2 K_{0}$, and $\beta=\omega$ if $g=0$. It will be shown later that $\beta$ plays a key role in two-photon QRMs. The second diagonal element is

$$
\begin{aligned}
H_{22}= & (2 \omega \cosh 2 r-4 g \sinh 2 r) K_{0} \\
& +(\omega \sinh 2 r-2 g \cosh 2 r)\left(K_{+}+K_{-}\right)-\frac{\epsilon+\omega}{2},
\end{aligned}
$$

and the squeezing parameter is

$$
r=\frac{1}{4} \ln \left(\frac{1-2 g / \omega}{1+2 g / \omega}\right)
$$

It is obvious that the coupling strength $g<\omega / 2$ leads to a real squeezing parameter.
Based on the squeezing transformation, we propose the corresponding wave function as

$$
\left|\Psi_{A}\right\rangle^{q}=\left(\begin{array}{l}
\sum_{m=0}^{\infty} \sqrt{\left[2\left(m+q-\frac{1}{4}\right)\right]} e_{m}^{(q)}|q, m\rangle_{A} \\
\sum_{m=0}^{\infty} \sqrt{\left[2\left(m+q-\frac{1}{4}\right)\right] !} f_{m}^{(q)}|q, m\rangle_{A},
\end{array}\right)
$$

where the new basis $|q, m\rangle_{A}=S_{A}|q, m\rangle$ with $|q, m\rangle$ as the Fock state. The coefficients $e_{m}^{(q)}$ and $f_{m}^{(q)}$ are to be determined in the following.

In the case of the Lie algebra considered here, $K_{0}|q, 0\rangle_{A}=$ $q|q, 0\rangle_{A}$ where $q=\frac{1}{4}$ and $\frac{3}{4}$ divide the whole Hilbert space $\mathcal{H}$ into even and odd sectors and label them, respectively. For the even subspace, $\mathcal{H}_{1 / 4}=\left\{a^{\dagger n}|0\rangle, n=0,2,4, \ldots\right\}$, and for the odd subspace, $\mathcal{H}_{3 / 4}=\left\{a^{\dagger n}|0\rangle, n=1,3,5, \ldots\right\}$, corresponding to even or odd Fock number basis. The Bargmann index $q$ allows us to deal with both cases independently.

The $\operatorname{su}(1,1)$ Lie algebra operators satisfy

$$
\begin{aligned}
K_{0}|q, n\rangle_{A} & =(n+q)|q, n\rangle_{A}, \\
K_{+}|q, n\rangle_{A} & =\sqrt{\left(n+q+\frac{3}{4}\right)\left(n+q+\frac{1}{4}\right)}|q, n+1\rangle_{A}, \\
K_{-}|q, n\rangle_{A} & =\sqrt{\left(n+q-\frac{3}{4}\right)\left(n+q-\frac{1}{4}\right)}|q, n-1\rangle_{A} .
\end{aligned}
$$

Projecting both sides of the Schrödinger equation onto $|q, n\rangle_{A}$ gives a linear relation between coefficients $e_{n}^{(q)}$ and $f_{n}^{(q)}$,

$$
e_{n}^{(q)}=\frac{\Delta / 2}{2 \beta(n+q)-E+\frac{\epsilon-\omega}{2}} f_{n}^{(q)},
$$

and a three-term linear recurrence relation is given by

$$
f_{n+1}^{(q)}=\frac{2\left(2 \omega^{2}-\beta^{2}\right)(n+q)-\beta\left(E+\frac{\epsilon+\omega}{2}\right)-\frac{\Delta^{2} \beta / 4}{2 \beta(n+q)-E+\frac{\epsilon-\omega}{2}}}{8 g \omega\left(n+q+\frac{1}{4}\right)\left(n+q+\frac{3}{4}\right)} f_{n}^{(q)}-\frac{1}{4\left(n+q+\frac{1}{4}\right)\left(n+q+\frac{3}{4}\right)} f_{n-1}^{(q)} .
$$

All coefficients $f_{n}^{(q)}$ and $e_{n}^{(q)}$ can be calculated with initial conditions $f_{-1}^{(q)}=0$ and $f_{0}^{(q)}=1$.

We further apply the second squeezing operator $S_{B}=e^{-(r / 2)\left(a^{2}-a^{\dagger 2}\right)}$ to the Hamiltonian (21) and suggest the wave function as

$$
\left|\Psi_{B}\right\rangle^{q}=\left(\begin{array}{l}
\sum_{m=0}^{\infty}(-1)^{m} \sqrt{\left[2\left(m+q-\frac{1}{4}\right)\right]} ! c_{m}^{(q)}|q, m\rangle_{B} \\
\sum_{m=0}^{\infty}(-1)^{m} \sqrt{\left[2\left(m+q-\frac{1}{4}\right)\right] !} d_{m}^{(q)}|q, m\rangle_{B},
\end{array}\right)
$$

where $|q, m\rangle_{B}=S_{B}|q, m\rangle$. Similarly, we can obtain a linear relation between the coefficients $c_{n}^{(q)}$ and $d_{n}^{(q)}$,

$$
d_{n}^{(q)}=\frac{\Delta / 2}{2 \beta(n+q)-E-\frac{\epsilon+\omega}{2}} c_{n}^{(q)},
$$

and the three-term linear recurrence relation is

$$
c_{n+1}^{(q)}=\frac{2\left(2 \omega^{2}-\beta^{2}\right)(n+q)-\beta\left(E-\frac{\epsilon-\omega}{2}\right)-\frac{\Delta^{2} \beta / 4}{2 \beta(n+q)-E-\frac{\epsilon+\omega}{2}}}{8 g \omega\left(n+q+\frac{1}{4}\right)\left(n+q+\frac{3}{4}\right)} c_{n}^{(q)}-\frac{1}{4\left(n+q+\frac{1}{4}\right)\left(n+q+\frac{3}{4}\right)} c_{n-1}^{(q)} .
$$

Left multiplying the vacuum state $\langle q, 0|$ to the extended squeezed state $|q, m\rangle_{A}$ and $|q, m\rangle_{B}$, we can obtain the inner product,

$$
\langle q, 0 \| q, m\rangle_{A}=\frac{(-\tanh r)^{m}}{\sqrt{\cosh r}} \frac{\sqrt{\left[2\left(m+q-\frac{1}{4}\right)\right] !}}{2^{m} m !}, \quad\langle q, 0 \| q, m\rangle_{B}=\frac{(\tanh r)^{m}}{\sqrt{\cosh r}} \frac{\sqrt{\left[2\left(m+q-\frac{1}{4}\right)\right] !}}{2^{m} m !} .
$$



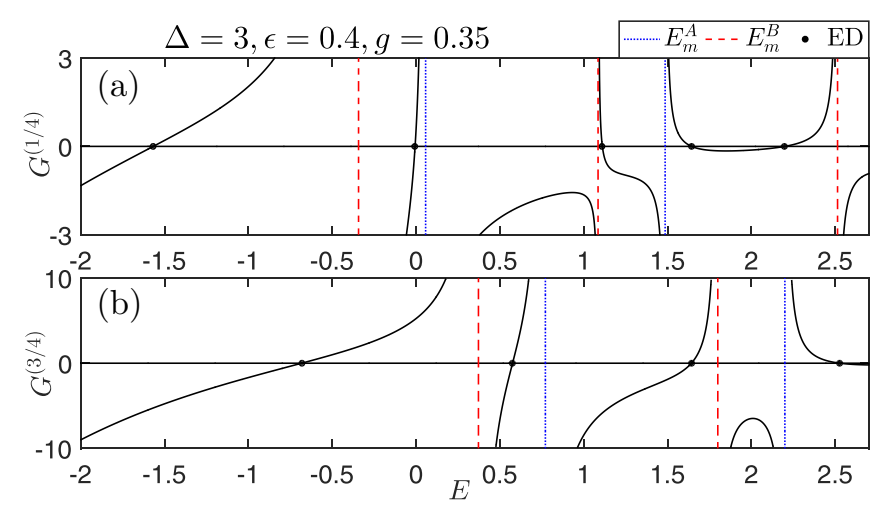

FIG. 3. $G$ curves for $\omega=1, \Delta=3, \epsilon=0.4, g=0.35 . q=$ $1 / 4$ (upper) and $q=3 / 4$ (lower). The blue dashed lines are $E_{m}^{A}$ in Eq. (33), and the red dashed lines are $E_{m}^{B}$ in Eq. (34), $m=0,1,2 \cdots$. The data by numerical diagonalizations are indicated by black dots, which agree excellently with the zeros of the $G$ functions (32).

If both wave-functions $\left|\Psi_{A}\right\rangle^{q}$ and $\left|\Psi_{B}\right\rangle^{q}$ for the same $q$ are the true eigenfunction for a nondegenerate eigenstate with eigenvalue $E$, they should be proportional with each other, i.e., $\left|\Psi_{A}\right\rangle^{q}=z\left|\Psi_{B}\right\rangle^{q}$, where $z$ is a complex constant. Projecting both sides of this identity onto the original vacuum state $|q, 0\rangle$, we, consequently, obtain the $G$ function of the asymmetric tpQRM as

$$
\begin{aligned}
G^{(q)}= & \left(\frac{\Delta}{2}\right)^{2}\left[\sum_{m=0}^{\infty} \frac{f_{m}^{(q)} \Omega_{m}^{(q)}}{2 \beta(m+q)+\frac{\epsilon-\omega}{2}-E}\right] \\
& \times\left[\sum_{m=0}^{\infty} \frac{c_{m}^{(q)} \Omega_{m}^{(q)}}{2 \beta(m+q)-\frac{\epsilon+\omega}{2}-E}\right] \\
& -\sum_{m=0}^{\infty} f_{m}^{(q)} \Omega_{m}^{(q)} \sum_{m=0}^{\infty} c_{m}^{(q)} \Omega_{m}^{(q)},
\end{aligned}
$$

where

$$
\Omega_{m}^{(q)}=\frac{(-\tanh r)^{m}}{\sqrt{\cosh r}} \frac{\left[2\left(m+q-\frac{1}{4}\right)\right] !}{2^{m} m !} .
$$

If set $\epsilon=0$, the $G$ function for the symmetric tpQRM [17] is recovered. The zeros of the $G^{(q)}$ function give the regular spectrum in the $q$ subspace of the asymmetric tpQRM.

One immediately finds that the $G$ function diverges at either

or

$$
E_{m}^{A}=2 \beta(m+q)+\frac{\epsilon-\omega}{2},
$$

$$
E_{m}^{B}=2 \beta(m+q)-\frac{\epsilon+\omega}{2},
$$

with $m=0,1,2, \ldots$. They are also labeled as two types $(A$ and $B)$ pole energies, similar to the asymmetric QRM.

$G$ curves at $q=1 / 4$ and $3 / 4$ for $\epsilon=0.4, g=0.35$, and $\Delta=3$ with $\omega=1$ are plotted in Fig. 3 . The zeros are easily detected. As usual, one can check it easily with numerics, and an excellent agreement can be achieved. The poles given in Eqs. (33) and (34) are marked with vertical lines. The $G$ curves indeed show diverging behavior when approaching the poles.

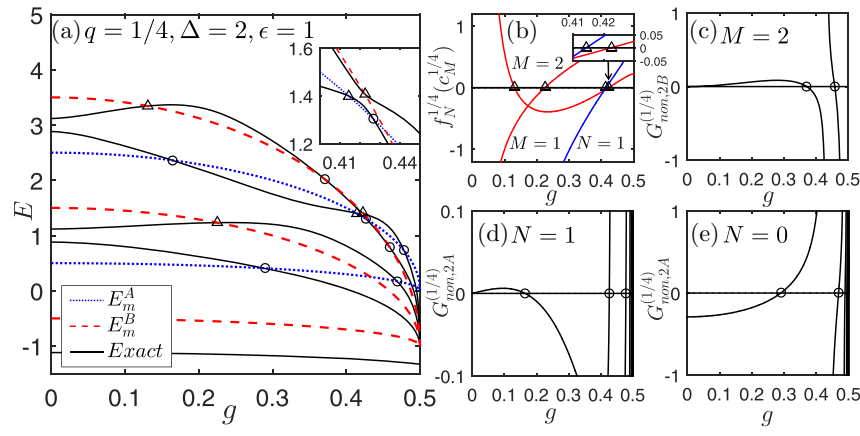

FIG. 4. (a) The spectrum for the first five levels and the nondegenerate exceptional solutions for the asymmetric tpQRM with $\omega=1, \Delta=2, \epsilon=1, q=1 / 4$. The blue dashed lines are $E_{m=0,1}^{A}$ by Eq. (33), and red dashed lines are $E_{m=0,1,2}^{B}$ by Eq. (34). The inset on an enlarged scale clearly displays an avoided crossing rather than a true level crossing. $f_{N=1}^{(1 / 4)}, c_{M=1,2}^{(1 / 4)}$ curves are exhibited in (b), whose zeros are indicated by open triangles, agreeing with the same symbols in the spectrum (a). The nondegenerate exceptional $G$ function $G_{\text {non }, 2 B}^{(1 / 4)}$ in Eq. (B9) for $M=2$ and $G_{\text {non, } 2 A}^{(1 / 4)}$ in Eq. (B8) for $N=1,0$ are given in (c)-(e), respectively. Their zeros are denoted by open circles, which are excellently consistent with the nondegenerate exceptional points indicated by the same symbols in the spectrum (a).

We next present the spectra in Fig. 4(a) for the parameters $q=1 / 4, \Delta=2, \epsilon=1$ with $\omega=1$. The crossing points of the energy levels and the pole lines (33) and (34), known as nondegenerate exceptional points, are marked with open symbols. In Appendix B 2, we derive the nondegenerate exceptional solutions analytically in details. All these nondegenerate exceptional points can be confirmed. The solutions by the coefficient polynomial equations $f_{N=1}^{1 / 4}=0$ and $c_{M=1,2}^{1 / 4}=0$ are indicated by open triangles in Fig. 4(b) and denoted with the same symbols in Fig. 4(a). Zeros of the nondegenerate exceptional $G$ functions (B8) and (B9) corresponding to open circles in Figs. 4(c)-4(e) are indicated by the open circles in Fig. 4(a).

As revealed on an enlarged scale in the inset of Figs. 4(a) and 4(b), two open triangles do not coincide, indicating an avoided crossing at the bias parameter $\epsilon=1$. We will show in the left panels of Fig. 5 at slightly larger bias $\epsilon=1.0954$ in the next section that the two open triangles also obtained from $f_{N=1}^{(1 / 4)}=0$ and $c_{M=2}^{(1 / 4)}=0$ can eventually meet. Thus, it should be very interesting to see how an avoided crossing essentially turns to a true level crossing when $\epsilon=1 \rightarrow 1.0954$.

\section{DOUBLY DEGENERATE STATES IN ASYMMETRIC TWO-PHOTON QRM}

The natural question arises what the condition is for the existence of the level crossings in the same $q$ subspace in the asymmetric tpQRM. According to the pole energies (33) and (34), if $E_{M}^{A}=E_{N}^{B}$, then

$$
\epsilon=2 \beta(M-N),
$$

the same pole energy takes

$$
E=(M+N+2 q) \beta-\frac{1}{2} \omega .
$$


Interestingly, Eq. (35) entails $\epsilon$ to be an even multiple of the renormalized cavity frequency $\beta$, in contrast to the asymmetric one-photon QRM where $\epsilon$ should be an multiple of the cavity frequency $\omega$ under the condition (13) for level crossings. It makes sense that only the two-photon process is involved in the two-photon model, whereas the single-photon process in the one-photon model.

Without loss of generality, we also only consider $M>N$ here. From Eqs. (27) and (30), one immediately note that the coefficient $e_{N}^{(q)}$ in Eq. (26) $\left[d_{M}^{(q)}\right.$ in Eq. (29)] would diverge at the same pole energy (36). Similar to the asymmetric QRM case, the series expansion coefficients in the wave-functions (25) and (28) should be analytic and vanish as or before $n \rightarrow$ $\infty$.

Regarding states with the energy (36), the numerator of the right-hand side of (26) and (29) should also vanish so that $e_{N}^{(q)}\left(d_{M}^{(q)}\right)$ remains finite, which requires

$$
f_{N}^{(q)}(M, g)=0 ; \quad c_{M}^{(q)}(N, g)=0 .
$$

Note that $f_{N}^{(q)}$ and $c_{M}^{(q)}$ can be, respectively, obtained from the recurrence relations (27) and (30) by using the same pole energy (36),

$$
\begin{aligned}
f_{n+1}^{(q)}= & \frac{2 \omega^{2}(n+q)-\beta^{2}(n+M+2 q)+\frac{\Delta^{2}}{16(N-n)}}{4 g \omega\left(n+q+\frac{1}{4}\right)\left(n+q+\frac{3}{4}\right)} f_{n}^{(q)} \\
& -\frac{1}{4\left(n+q+\frac{1}{4}\right)\left(n+q+\frac{3}{4}\right)} f_{n-1}^{(q)}, \\
c_{n+1}^{(q)}= & \frac{2 \omega^{2}(n+q)-\beta^{2}(n+N+2 q)+\frac{\Delta^{2}}{16(M-n)}}{4 g \omega\left(n+q+\frac{1}{4}\right)\left(n+q+\frac{3}{4}\right)} c_{n}^{(q)} \\
& -\frac{1}{4\left(n+q+\frac{1}{4}\right)\left(n+q+\frac{3}{4}\right)} c_{n-1}^{(q)} .
\end{aligned}
$$

Similar to the asymmetric one-photon QRM, we conjecture that both $f_{N}^{(q)}(M, g)=0$ and $c_{M}^{(q)}(N, g)=0$ could give the same positive real $g$ and $\epsilon$ under the constrained condition (35), leading to levels crossing at the same pole energy. Although it would be interesting to rigorously prove the conjecture in the two-photon case mathematically, we also confine us here to an analytical closed-form proof only for small values of $N$ and $M$ and numerically confirmation for large $N$ and $M$ in searching for physically reasonable coupling strength $g$. Similar to the asymmetric QRM, we analytically prove in Appendix A 2 that for some small values of $N$ and $M$ both $f_{N}^{(q)}(M, g)=0$ and $c_{M}^{(q)}(N, g)=0$ in Eq. (37) give the same values for $\epsilon$ and $g$. Two energy levels cross the corresponding pole lines at the same values of $\epsilon$ and $g$ where the two pole lines also cross. Thus true level crossings also happen in the asymmetric tpQRM. Compared to the one-photon QRM where $\epsilon$ can be determined independently, in the asymmetric tpQRM, we need to solve Eqs. (35) and (37) simultaneously to determine $\epsilon$ and $g$.

To illustrate the level crossing clearly, we show the energy spectra of the asymmetric tpQRM at $N=1, \Delta=2$ with $\omega=$ 1 for $q=1 / 4, M=2$ (left), $q=1 / 4, M=3$ (middle), and $q=3 / 4, M=3$ (right) in the low panels of Fig. 5. The corresponding values of $\epsilon$ are just those determined by Eq. (A9), which, in turn, are $\epsilon=1.0954,1.8516$, and 2.49442 from

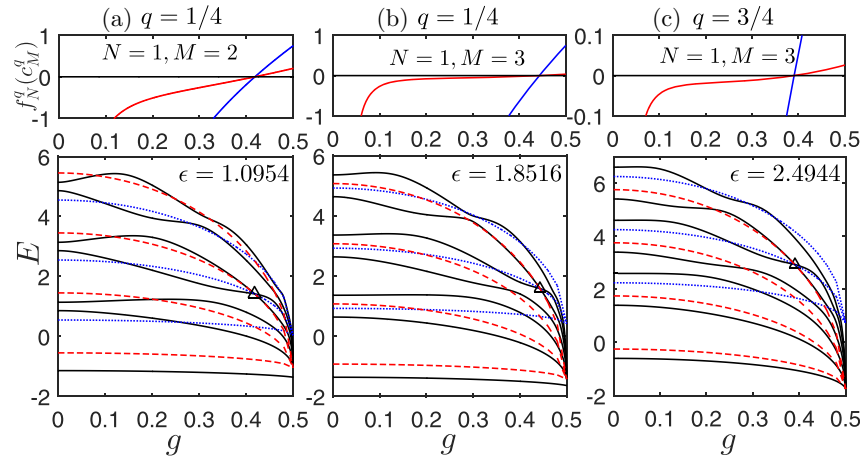

FIG. 5. Energy spectra for $\Delta=2$ and $\omega=1$ are plotted in the lower panels: $(q, \epsilon)=(1 / 4,1.0954)$ (left), $(1 / 4,1.8516)$ (middle), and $(3 / 4,2.4944)$ (right). The black lines are energy levels, the blue dashed lines are $E_{m}^{A}$, and the red dashed lines are $E_{m}^{B}$. Open triangles indicate the doubly degenerate level crossings. $f_{N=1}^{(q)}(M, g)$ (blue) and $c_{M}^{(q)}(N=1, g)$ (red) curves are displayed in the upper panels just on the top of the corresponding spectra. Zeros are the same for both curves, indicating true level crossings.

left to right. Interestingly, the level crossing point indicated by the open triangle really appears in each spectrum, confirming the analytical prediction.

The upper panels in Fig. 5 present the curves for $f_{1}^{(q)}(M, g)$ and $c_{M}^{(q)}(1, g)$. It is clear that the zeros of both functions are the same and are consistent with the coupling strength at the level crossing points. For example, for $q=1 / 4, \Delta=$ 2, $M=2$ two energy levels cross exactly at $g=0.4183$ by Eq. (A10). This analytical finding is in excellent consistent with numerical results presented in the left panels of Fig. 5. This agreement also applies to the middle and right panels. As expected, the type- $A$ pole lines $E_{N=1}^{A}$ and the type- $B$ pole lines $E_{M}^{B}$ also cross at the degenerate points in the low panels of Fig. 5.

For $N=1$, no matter what is the value of $M>N$, from Eq. (A7), we can, at most, find one solution for $g$. By contrast, for $N>1, f_{N}^{(q)}(M, g)=0$ is a polynomial equation with higher-order terms, which would result in more than one solution for $g$.

To demonstrate the true level crossings for $N>1$, we plot $f_{N}^{(q)}(M, g)$ and $c_{M}^{(q)}(N, g)$ curves with $N=2$ and $M=3$ for $\Delta=2, \omega=1, q=1 / 4$ in Fig. 6(a). It is to note that zeros of both curves yield the same solutions $g_{1,2}=0.3015,0.4686$ predicted by Eq. (A11), and two values of biases $\epsilon_{1,2}=$ $1.5954,0.6974$ are determined accordingly. We then display the energy spectra for these two values of $\epsilon$ in Figs. 6(b) and 6(c) for the same model parameters, respectively. The level crossings are clearly revealed at the analytical predicted coupling strength. In other words, the $N=2$ type- $A$ pole line and the $M=3$ type- $B$ pole line indeed cross at the same doubly degenerate points.

Finally, the doubly degenerate states at the true level crossing points can be expressed explicitly in terms of the BOA as

$$
\left|\Psi_{A}\right\rangle^{q}=\left(\begin{array}{l}
\sum_{m=0}^{N} \sqrt{\left[2\left(m+q-\frac{1}{4}\right)\right] !} e_{m}^{(q)}|q, m\rangle_{A} \\
\sum_{m=0}^{N-1} \sqrt{\left[2\left(m+q-\frac{1}{4}\right)\right] !} f_{m}^{(q)}|q, m\rangle_{A},
\end{array}\right)
$$



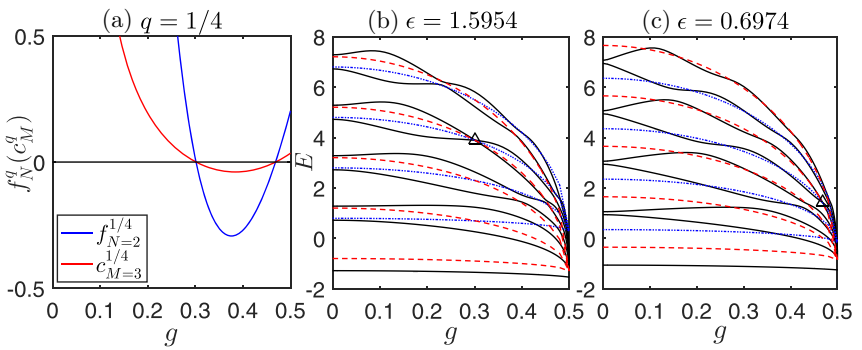

FIG. 6. $f_{N=2}^{(1 / 4)}$ and $c_{M=3}^{(1 / 4)}$ curves for $\Delta=2, \omega=1$, and $q=1 / 4$ are exhibited in (a). The zeros are the same for both curves. The energy spectra for same parameters are presented in (b) for $\epsilon=$ 1.5954 and (c) $\epsilon=0.6974$. The black lines are energy levels, the blue dashed lines are $E_{m}^{A}$, and the red dashed lines are $E_{m}^{B}$. Two open triangles indicate the doubly degenerate level crossing points, and the locations are consistent with two zeros in (a).

and

$$
\left|\Psi_{B}\right\rangle^{q}=\left(\begin{array}{l}
\sum_{m=0}^{M-1}(-1)^{m} \sqrt{\left[2\left(m+q-\frac{1}{4}\right)\right]} ! c_{m}^{(q)}|q, m\rangle_{B} \\
\sum_{m=0}^{M}(-1)^{m} \sqrt{\left[2\left(m+q-\frac{1}{4}\right)\right] !} d_{m}^{(q)}|q, m\rangle_{B},
\end{array}\right)
$$

respectively, where $e_{N}^{(q)}$ and $d_{M}^{(q)}$ are given by Eqs. (B5) and (B7). Because these two wave functions are not obtained from the $G$ function based on the proportionality, so they are different, leading to doubly degenerate states. Both wave functions terminate at finite terms, so they are the quasiexact solutions of the asymmetric tpQRM.

\section{DISCUSSIONS}

From the spectra in Figs. 5 and 6, one might speculate that level crossings seldom happen in the asymmetric tpQRM. Actually it is not that case. If we always incorporate Eq. (35) required by the level crossings, we may acquire similar spectra graphs as Figs. 1 and 2 in the one-photon case. In doing so, we calculate the energy as a function of $g$, and at the same time, $\epsilon$ also changes as Eq. (35). To display the level crossings in asymmetric tpQRM more distinctly, we can make the pole lines horizontal, thus, we plot the normalized energy $E^{\prime}=\frac{E+\omega / 2}{2 \beta}-q+\frac{\epsilon}{4 \beta}$ as a function of $g$ and simultaneously varying $\epsilon=k \beta$ in Fig. 7 for $k=0,1,2$, and 4 at $\Delta=2$ with $\omega=1, q=1 / 4$.

When $\epsilon$ is an even multiple of the normalized cavity frequency $\beta$ entailed in Eq. (35), i.e., $k$ is an even integer including the symmetric case $k=0$, we find that the two equations in Eq. (37) result in the same positive solutions for the coupling strength as indicated with open triangles in Figs. 7(a), 7(c), and 7(d). One can note that the level crossings happen regularly. The crossing points at the $N=1$ type- $A$ pole line in Figs. 7(c) and 7(d) are just corresponding to those in Figs. 5(a) and 5(b), whereas the two crossing points at the $N=2$ type- $A$ pole line in Fig. 7(c) are corresponding to those in Fig. 6.

However, if $k$ is not an even integer, no level crossings happen, and a lot of nondegenerate exceptional points emerges instead. As exhibited in Fig. 7(b) for $k=1$, the open triangles correspond to the nondegenerate exceptional points by (a) $\epsilon=0$
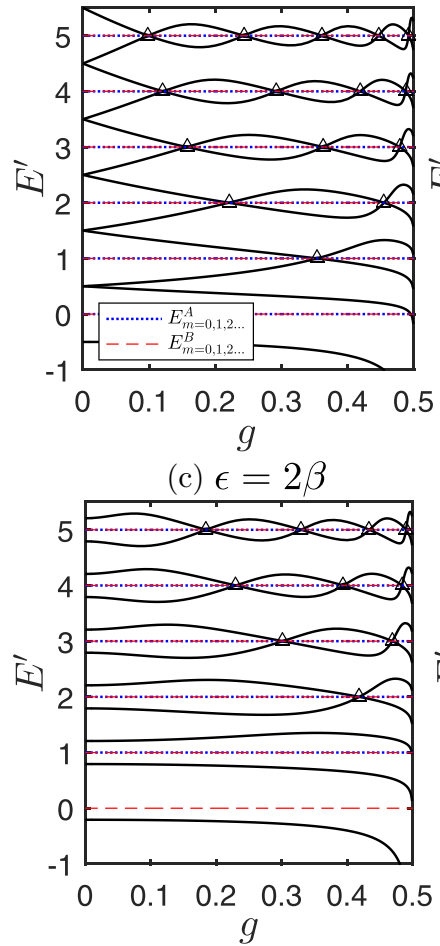

(c) $\epsilon=2 \beta$ (b) $\epsilon=\beta$

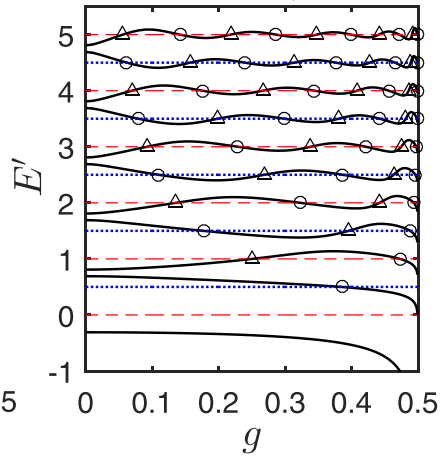

(d) $\epsilon=4 \beta$

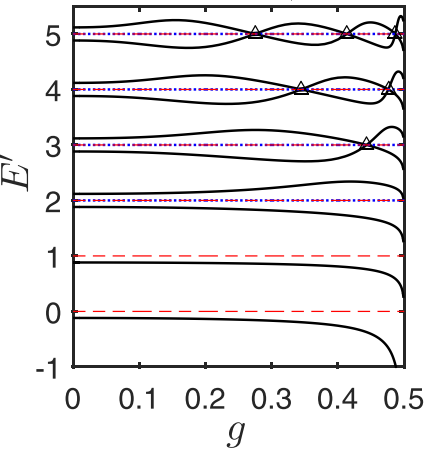

FIG. 7. The energy spectra $E^{\prime}=\frac{E+1 / 2}{2 \beta}-q+\frac{\epsilon}{4 \beta}$ for $\omega=$ 1, $\Delta=2, q=1 / 4, \epsilon=k \beta$ in $k=$ (a) 0 , (b) 1 , (c) 2 , and (d) 4 . Note particularly that $\epsilon$ changes with $g$ along the $g$ axis. The horizontal blue dotted lines correspond to the pole energy ones for $E_{N}^{A}$ and the red dashed lines to $E_{M}^{B}$. Only the overlapped pole lines with $N>0$ allow for the true level crossings. The triangles denotes the doubly degenerate crossing points in (a), (c), and (d). In (b), the triangles are obtained from $f_{N}^{(q)}=0$ and $c_{M}^{(q)}=0$ or, equivalently, from Eqs. (B4) and (B6), whereas open circles from Eqs. (B8) and (B9), all of them correspond to nondegenerate exceptional points.

Eqs. (B4) or (B6), whereas the open circles correspond to those by Eqs. (B8) and (B9).

We can also estimate the number of the doubly degenerate crossing points associated with the given $N$ type- $A$ pole line. For any $M>N$, generally, there are around $N$ crossing points due to the polynomial equation with $N$ terms $f_{N}^{(q)}(M, g)=0$ in Eq. (37), the detailed polynomial equations are derived from Eq. (38). This is to say, associated with the $N$ type- $A$ pole line, we generally have around $N$ degenerate crossing points for both asymmetric one-photon and two-photon QRMs. In Appendix A 3, we have employed the constrained conditions in the asymmetric both one- and two-photon QRMs and numerically found that they have nearly the same numbers of level crossing points in the range of integers of $N$ and $M$ in each case. Therefore, we could reach a conclusion that the number of the doubly degenerate crossing points in asymmetric tpQRM would be twice that in the asymmetric QRM due to two Bargmann indices in the former model.

Very interestingly, the efforts to look for the hidden symmetry responsible for the double degeneracy at the level crossing points for integer $\epsilon / \omega$ in the asymmetric one-photon QRM have been attempted recently $[33,39,44,45]$ and con- 
tinue to be a great interest. Since the doubly degenerate states within the same $q$ subspace also exist in the asymmetric tpQRM without any obvious symmetry, it should also be owing to the unknown hidden symmetry. Then an interesting question is what symmetry operators might exist in the asymmetric tpQRM and, hence, could account for the observed energy-level crossings. To construct the $k$ th hidden symmetry operator $J_{k}$ in the asymmetric tpQRM, the condition for the existence of the true level crossings $\epsilon=2 k \beta$ should be considered. Inspired by the parity operator in the symmetric QRMs, we speculate that the symmetry operators in the asymmetric tpQRM would take the form of $2 \times 2$ matrices of polynomials in operators $K_{0}, K_{ \pm}$in the usual $s u(1,1)$ Lie algebra and satisfy the commutation relation $\left[J_{k}, H_{2}^{\epsilon=k \beta}\right]=0$. It should be very interesting to rigorously find the general hidden symmetry operators in the future.

Such degeneracies due to hidden symmetries can be easily tested in superconducting qubit setups by measured transmission magnitude of the coupled qubit-cavity system if the external parameters meet the condition of the level crossings. Some avoided-level crossings could be driven to the true level crossings in the spectrum (cf. Fig. 3 of Ref. [5]). More interestingly, all properties described in quantum optics [3] where the static bias is usually lacking would appear in the superconducting qubit setups with the special bias supporting the double degeneracy. It would be particularly worthwhile with regard to investigating topological properties [44] if the conical intersection structure in the energy spectrum can be recovered.

\section{CONCLUSION}

In this paper, we have studied both the asymmetric QRM and the asymmetric tpQRM by the BOA in a unified way. The previously observed level crossing when the bias parameter $\epsilon$ is a multiple of cavity frequency in the asymmetric QRM is illustrated by a closed-from proof for low orders of the constrained polynomial equations in a transparent manner. For the asymmetric tpQRM, we find that the true level crossings can also happen in the same $q$ subspace if the qubit bias parameter $\epsilon$ is an even multiple of the $g$-dependent renormalized cavity frequency. We argue that the even multiple is originated from the two-photon process involved in the two-photon model. The doubly degenerate points can be also located analytically, similar to the asymmetric QRM. The number of the doubly degenerate points within the same subspace in the asymmetric tpQRM should be comparable with that in asymmetric QRM. The subspace of the asymmetric tpQRM possesses not any explicit symmetry, the newly found double degeneracy at the level crossings points, thus, also implies the hidden symmetry.

The hidden symmetry in the asymmetric QRM could be identified at the same integer $\epsilon / \omega$, whereas in the asymmetric tpQRM at the same integer $\epsilon /(2 \beta)$. The latter constraint on the parameter space for the occurrence of the double degeneracy is illuminating in searching for symmetry operators in the two-photon case. The present results may shed some light on the different nature of the hidden symmetries in the two asymmetric QRMs.

\section{ACKNOWLEDGMENT}

We acknowledge useful discussions with Daniel Braak. This work was supported by the National Science Foundation of China under Grant No. 11834005 and the National Key Research and Development Program of China under Grant No. 2017YFA0303002.

\section{APPENDIX A: DEMONSTRATION FOR THE SAME PHYSICAL SOLUTIONS OF THE TWO EQUATIONS IN THE CONSTRAINED CONDITIONS IN TWO ASYMMETRIC QRMS}

In this Appendix, we first present a closed-form proof for the conjecture that $f_{N}(M, g)=0$ and $c_{M}(N, g)=0$ in Eq. (15) could give the same real and positive solutions for the coupling strength $g$ with small numbers of $N$ and $M$ in the asymmetric one-photon QRM. In parallel, we then provide a closed-form proof for the conjecture that $f_{N}^{(q)}(M, g)=0$ and $c_{M}^{(q)}(N, g)=0$ in Eq. (37) could give the same real and positive solutions for the coupling strength $g$ with small numbers of $N$ and $M$ in the asymmetric tpQRM. Finally, we provide numerical confirmations on the conjecture with large range of integers $N$ and $M$ in two asymmetric QRMs. We set $\omega=1$ in both models for simplicity in this Appendix.

\section{Analytical proof for the small order of the constrained conditions in the asymmetric one-photon QRM}

Since $f_{0}=1$, we begin with the $N=1$ type- $A$ pole energy, and Eq. (16) becomes

$$
f_{1}(M, g)=\frac{1}{2 g}\left(4 g^{2}+\frac{1}{4} \Delta^{2}-M\right),
$$

its zero is simply

$$
g=\frac{1}{2} \sqrt{M-\left(\frac{\Delta}{2}\right)^{2}}
$$

which is dependent on $M$. If $\Delta>2 \sqrt{M}$, no real solution exists, so the level crossing does not occur along the $N=1$ pole line.

If we set $M=2$, i.e., $\epsilon=1$, we have

$$
g=\frac{1}{2} \sqrt{2-\left(\frac{\Delta}{2}\right)^{2}} .
$$

The second equation in Eq. (15) $c_{2}(1, g)=0$ yields

$$
\left(4 g^{2}+\frac{\Delta^{2}}{4}\right)\left(4 g^{2}+\frac{\Delta^{2}}{8}-1\right)-4 g^{2}=0,
$$

resulting in

$$
g=\frac{1}{2} \sqrt{2-\left(\frac{\Delta}{2}\right)^{2}},
$$

which is exactly the same as Eq. (A2), the solution for $f_{1}(2, g)=0$. It follows that two energy levels intersect with the same pole line at the same coupling strength $g$ in the spectra, indicating a true energy-level crossing. 
For $N=2$ type- $A$ pole energy, the first equation in Eq. (15) $f_{2}(M, g)=0$ becomes (we set $x=4 g^{2}$ for simplicity)

$$
\left(x+\frac{1}{4} \Delta^{2}-M+1\right)\left(x+\frac{1}{8} \Delta^{2}-M\right)-x=0,
$$

yielding

$$
x=\left(M-\frac{3}{16} \Delta^{2}\right) \pm \sqrt{\left(\frac{\Delta^{2}}{16}-1\right)^{2}+(M-1)} .
$$

If $M=3$, i.e., $\epsilon$ is still 1 , the solutions then read

$$
g=\frac{1}{8} \sqrt{-3\left(\Delta^{2}-16\right) \pm \sqrt{\left(\Delta^{2}-16\right)^{2}+512}} .
$$

On the other hand, the second equation in Eq. (15) $c_{3}(2, g)=$ 0 is

$$
\begin{aligned}
& \left(x+\frac{\Delta^{2}}{4}\right)\left[\left(x+\frac{\Delta^{2}}{8}-1\right)\left(x+\frac{\Delta^{2}}{12}-2\right)-x\right] \\
& -2 x\left(x+\frac{\Delta^{2}}{12}-2\right)=0,
\end{aligned}
$$

which interestingly gives the same solutions as in Eq. (A4), consistent with the conjecture. Here an unphysical solution $x=-\frac{1}{12} \Delta^{2}$ is omitted.

Next, we set $N=1, M=3$, thus, $\epsilon=2 . f_{1}(3, g)=0$ gives

$$
g=\frac{1}{4} \sqrt{12-\Delta^{2}} .
$$

By $c_{3}(1, g)=0$, we have

$$
\begin{aligned}
& \left(x+\frac{1}{4} \Delta^{2}+1\right)\left[\left(x+\frac{1}{8} \Delta^{2}\right)\left(x+\frac{1}{12} \Delta^{2}-1\right)-x\right] \\
& -2 x\left(x+\frac{1}{12} \Delta^{2}-1\right)=0 .
\end{aligned}
$$

Its solutions are

$$
x=3-\frac{1}{4} \Delta^{2} ; \quad x=-\frac{\Delta^{2}}{48}\left(5 \pm \sqrt{1-\frac{96}{\Delta^{2}}}\right) .
$$

Note that the second root is not a positive real value and is so omitted. The first root gives exactly the same $g$ in Eq. (A6).

\section{Analytical proof for the small order of the constrained conditions in the asymmetric tpQRM}

In this subsection, we turn to the a closed-form proof for the conjecture in the asymmetric tpQRM.

For the most simply case, we set $N=1$, then $f_{1}^{(q)}(M, g)=$ 0 gives

$$
4 q-(2 M+4 q) \beta^{2}+\frac{\Delta^{2}}{8}=0,
$$

then the location of the degenerate point is obtained

$$
\beta^{2}=\frac{2 q+\Delta^{2} / 16}{M+2 q},
$$

which is dependent on $M$. Also note that the positive real solution only exists for $\Delta<4 \sqrt{M}$. Subject to the constrained condition (35), we have

$$
\epsilon=(M-1) \sqrt{\frac{8 q+\Delta^{2} / 4}{2 q+M}} .
$$

If set $M=2, c_{2}^{(q)}(1, g)=0$ gives

$$
\begin{aligned}
& {\left[4(q+1)\left(1-\beta^{2}\right)+\frac{1}{8} \Delta^{2}\right]} \\
& \quad \times\left[2(2 q+1)\left(1-\beta^{2}\right)+\frac{1}{16} \Delta^{2}-2\right] \\
& \quad-4\left(q+\frac{1}{4}\right)\left(q+\frac{3}{4}\right)\left(1-\beta^{2}\right) \\
& \quad=0,
\end{aligned}
$$

we then have

$$
\beta^{2}=\frac{2 q+\Delta^{2} / 16}{2+2 q},
$$

which is the same as that in Eq. (A8) for $M=2$, consistent with our conjecture.

Next, we set $N=2, M=3 \cdot f_{2}^{(q)}(3, g)=0$ gives

$$
\begin{aligned}
& {\left[4\left(1-\beta^{2}\right)(2+q)+\frac{\Delta^{2}}{8}-4\right]} \\
& \quad \times\left[2\left(1-\beta^{2}\right)(3+2 q)+\frac{\Delta^{2}}{16}-6\right] \\
& \quad-4\left(1-\beta^{2}\right)\left(q+\frac{1}{4}\right)\left(q+\frac{3}{4}\right) \\
& =0 .
\end{aligned}
$$

The solutions at $q=\frac{1}{4}$ are

$$
\beta^{2}=\frac{1}{3}+\frac{23 \Delta^{2}}{2016} \pm \frac{1}{126} \sqrt{\frac{25 \Delta^{4}}{256}+\frac{21}{2} \Delta^{2}+1008,}
$$

and at $q=\frac{3}{4}$ are

$$
\beta^{2}=\frac{5}{11}+\frac{29 \Delta^{2}}{3168} \pm \sqrt{\frac{20}{363}+\frac{\Delta^{2}}{8712}+\frac{49 \Delta^{4}}{3168^{2}}},
$$

whereas $c_{3}^{(q)}(2, g)=0$ results in

$$
\begin{aligned}
& \left\{\left(2+q+\frac{\Delta^{2}}{32\left(1-\beta^{2}\right)}\right)\left[2\left(1-\beta^{2}\right)(3+2 q)+\frac{\Delta^{2}}{16}-2\right]\right. \\
& \left.\quad-\left(q+\frac{5}{4}\right)\left(q+\frac{7}{4}\right)\right\}\left[4\left(1-\beta^{2}\right)(1+q)+\frac{\Delta^{2}}{24}-4\right] \\
& \quad-\left[4\left(1-\beta^{2}\right)(2+q)+\frac{\Delta^{2}}{8}\right]\left(q+\frac{1}{4}\right)\left(q+\frac{3}{4}\right) \\
& =0 .
\end{aligned}
$$

If $q=\frac{1}{4}$, the solutions are

$$
\begin{aligned}
& \beta^{2}=\frac{1}{3}+\frac{23 \Delta^{2}}{2016} \pm \frac{1}{126} \sqrt{\frac{25 \Delta^{4}}{256}+\frac{21}{2} \Delta^{2}+1008}, \\
& \beta_{3}^{2}=1+\frac{\Delta^{2}}{120} .
\end{aligned}
$$

If $q=\frac{3}{4}$, the solutions are

$$
\begin{aligned}
& \beta^{2}=\frac{5}{11}+\frac{29 \Delta^{2}}{3168} \pm \sqrt{\frac{20}{363}+\frac{\Delta^{2}}{8712}+\frac{49 \Delta^{4}}{3168^{2}}}, \\
& \beta_{3}^{2}=1+\frac{\Delta^{2}}{168}
\end{aligned}
$$


Omitting the unreasonable solutions $\beta_{3}$, we can find that both $f_{2}^{(q)}(3, g)=0$ and $c_{3}^{(q)}(2, g)$ give the same crossing coupling strengths for $q=1 / 4$ and $3 / 4$, respectively,

$$
g_{1,2}^{(1 / 4)}=\frac{1}{2} \sqrt{\frac{2}{3}-\frac{23}{2016} \Delta^{2} \pm \frac{\sqrt{25 \Delta^{4}+2688 \Delta^{2}+258048}}{2016}},
$$

$$
g_{1,2}^{(3 / 4)}=\frac{1}{2} \sqrt{\frac{6}{11}-\frac{29}{3168} \Delta^{2} \pm \frac{\sqrt{49 \Delta^{4}+1152 \Delta^{2}+552960}}{3168}} .
$$

which also agree well with our conjecture.

\section{Numerical confirmation for the two conjectures in both asymmetric QRMs}

In this subsection, we extensively demonstrate that for large $N$ and $M(>N)$, the two equations in either Eq. (15) or Eq. (37) give the same physics solutions in both asymmetric QRMs. We sets $N$ from 1 to 10 and $M$ from 2 to 20 for both one-photon QRM and one-photon tpQRM in the $q=1 / 4$ subspace at $\Delta=2$. First, we find that physics solutions from $f_{N}=0$ and $c_{M}=0$ are exactly the same in either case, confirming the conjectures numerically. Second, there are 715 level crossings points for both cases, indicating $N$ roots in the $N$-order polynomial equations in both models at $\Delta=2$. Generally, the root number is equal to or slightly less than $N$ for any $\Delta$. This is to say, for any values of $\Delta$, the numbers of the level crossings are generally nearly the same for the same ranges of $N$ and $M$ in asymmetric QRMs.

In Fig. 8, the doubly degenerate level crossing points are visualized in a three-dimensional (3D) view in $(\epsilon, g, E)$ space for the asymmetric one-photon QRM and in $(\epsilon / 2 \beta, g, E)$ space for the asymmetric tpQRM at $\Delta=2$. It is interesting to draw planes for level crossings in both cases as $\epsilon$ is simply scaled by a $g$-dependent factor $2 \beta$ in the two-photon case. In the original $3 \mathrm{D}(\epsilon, g, E)$ space, all the degenerate crossing points in asymmetric QRM are confined on equally spaced integer $\epsilon / \omega$ planes, whereas those in asymmetric tpQRM are actually locked in different cylindrical surfaces with integer $\epsilon /(2 \beta)$. Those different constrained surfaces in the model parameter spaces for the occurrence of the double degeneracy in two models should be considered in the definition of conserved operators and the detection of hidden symmetries.

\section{APPENDIX B: NONDEGENERATE EXCEPTIONAL SOLUTIONS}

In this Appendix, we derive the nondegenerate exceptional solutions in both asymmetric one-photon QRM and asymmetric tpQRM, respectively, in the following two subsections. In the asymmetric models, the number of pole lines are doubled compared to the corresponding symmetric models, cf. Eqs. (11) and (12), so the nondegenerate exceptional points appear more easily.

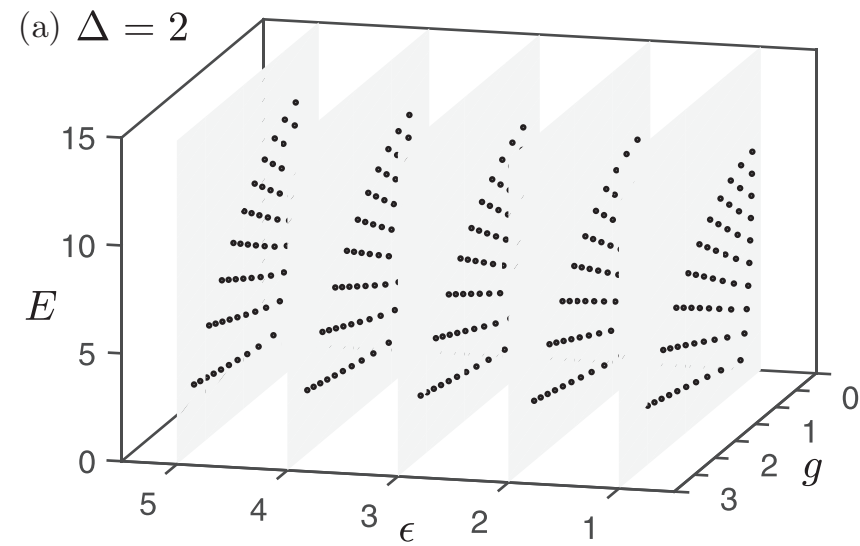

(b) $q=1 / 4, \Delta=2$

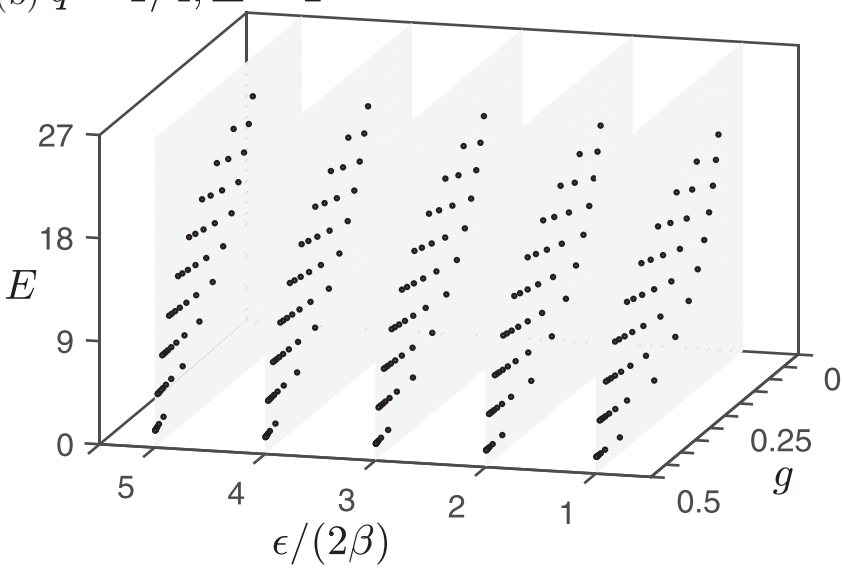

FIG. 8. Three-dimensional view for the doubly degenerate level crossing points at $\omega=1, \Delta=2$ for (a) the asymmetric one-photon QRM and (b) tpQRM in the $q=1 / 4$ subspace. We set $N$ from 1 to 10 and $M$ from 2 to 20 for both cases, and the numbers of the true level crossing points are the same. The data are drawn from part of the level crossings in this range.

\section{The asymmetric one-photon QRM}

In principle, all nondegenerate states including nondegenerate exceptional ones can be obtained by the $G$-function (10) because it is built based on the proportionality (9), only excluding the degenerate states. These states have been first analyzed for the symmetric QRM with the Bargmann space technique in Ref. [19] and later in Refs. [41,46,47]. We believe that the BOA has advantages with regard to the nondegenerate exceptional solutions, which cannot be found with any ansatz.

Note from $G$-function (10) that at the pole energy either (11) or (12) the denominator of the associated term becomes zero so this term would diverge and should be treated specially. For a physics state to avoid the divergence, the numerator $f_{N}(g)$ or $c_{M}(g)$ should also vanish. It is very important to see that $f_{N}(g)$ or $c_{M}(g)$ could vanish in two different ways. First, $f_{n}\left(c_{m}\right)$ can be obtained by using the three-term recurrence relations (6) and (8) from $f_{0}=1\left(c_{0}=\right.$ $1)$ and $f_{N}=0\left(c_{M}=0\right)$ until $n=N(m=M)$. Second, one can set $f_{n \leqslant N}=0\left(c_{m \leqslant M}=0\right)$ and $e_{n=N}=1\left(d_{m=M}=1\right)$ at the beginning directly and obtain all remaining coefficients by the recurrence relation (6) and (8). This is to say, we 
have two ways to overcome the divergence. In the infinite summation where the diverging term is present, we may cut off all the terms either after or before this diverging one. E.g., for the $N$ th type- $A$ pole line, we may terminate the infinite summation at the diverging term following the same idea outlined in the last section for the degenerate states. So the first nondegenerate exceptional $G$ function can be written as

$$
\begin{aligned}
G_{1 p}^{\mathrm{non}, 1 A}= & {\left[\sum_{n=0}^{N-1} \frac{\Delta f_{n}}{2 \omega(n-N)}\left(\frac{g}{\omega}\right)^{n}+e_{N}\left(\frac{g}{\omega}\right)^{N}\right] } \\
& \times\left[\sum_{n=0}^{\infty} \frac{\Delta c_{n}}{2\left(n \omega-g^{2} / \omega-\epsilon\right)}\left(\frac{g}{\omega}\right)^{n}\right] \\
& -\sum_{n=0}^{N-1} f_{n}\left(\frac{g}{\omega}\right)^{n} \sum_{n=0}^{\infty} c_{n}\left(\frac{g}{\omega}\right)^{n} \\
= & 0,
\end{aligned}
$$

where $e_{N}$ is given by Eq. (18). Note that the remaining terms vanish because all coefficients become zero. We can also remove all terms before the diverging term in the summation and give the second nondegenerate exceptional $G$ function as

$$
\begin{aligned}
G_{1 p}^{\mathrm{non}, 2 A}= & {\left[\left(\frac{g}{\omega}\right)^{N}+\sum_{n=N+1}^{\infty} \frac{\Delta f_{n}}{2 \omega(n-N)}\left(\frac{g}{\omega}\right)^{n}\right] } \\
& \times\left[\sum_{n=0}^{\infty} \frac{\Delta c_{n}}{2\left(n \omega-g^{2} / \omega-\epsilon\right)}\left(\frac{g}{\omega}\right)^{n}\right] \\
& -\sum_{n=N+1}^{\infty} f_{n}\left(\frac{g}{\omega}\right)^{n} \sum_{n=0}^{\infty} c_{n}\left(\frac{g}{\omega}\right)^{n} \\
= & 0,
\end{aligned}
$$

with the initial condition $e_{N}=1$. The nondegenerate exceptional $G$-functions $G_{1 p}^{\text {non,1B }}$ and $G_{1 p}^{\text {non, } 2 B}$ associated with the type- $B$ pole line can be obtained similarly by modifying the other infinite summation, which are not shown here.

Two nondegenerate exceptional $G$-functions (B1) and (B2) provide different exceptional solutions, which comprise the full nondegenerate exceptional points associated with the type- $A$ pole lines. Particularly, $f_{N}=0$ or $c_{M}=0$ is implied Eq. (B1) or $G_{1 p}^{\text {non, } 1 B}=0$, thus, can be also used to give the same nondegenerate exceptional points in a simpler way. Just as pointed out in Ref. [24], for noninteger $\epsilon$, a subset of the nondegenerate exceptional points associated with the pole lines can be given by the vanishing coefficients $f_{m}$ or $c_{m}$, equivalently, using Eq. (B1) or $G_{1 p}^{\text {non }, 1 B}=0$ here. However, Eq. (B1) and $G_{1 p}^{\text {non, } 1 B}=0$ fail at integer $\epsilon$ including $\epsilon=0$ because $f_{N}=0$ or $c_{M}=0$ actually results in the doubly degenerate states, which results in nonzero $G$ function in this case.

Interestingly, for integer $\epsilon$, two types of pole line may merge together. In this case, besides the doubly degenerate points, the nondegenerate exceptional points can additionally be generated by the intersection with one energy level alone. At the same pole energy (14), the second nonde- generate exceptional $G$-function Eq. (B2) would be further modified as

$$
\begin{aligned}
G_{1 p}^{\mathrm{non}}= & {\left[\left(\frac{g}{\omega}\right)^{N}+\sum_{n=N+1}^{\infty} \frac{\Delta f_{n}}{2 \omega(n-N)}\left(\frac{g}{\omega}\right)^{n}\right] } \\
& \times\left[\left(\frac{g}{\omega}\right)^{M}+\sum_{n=M+1}^{\infty} \frac{\Delta c_{n}}{2 \omega(n-M)}\left(\frac{g}{\omega}\right)^{n}\right] \\
& -\sum_{n=N+1}^{\infty} f_{n}\left(\frac{g}{\omega}\right)^{n} \sum_{n=M+1}^{\infty} c_{n}\left(\frac{g}{\omega}\right)^{n} \\
= & 0,
\end{aligned}
$$

where $e_{n<N}=0, e_{N}=1$ and $d_{n<M}=0, d_{M}=1$, the other coefficients can still be obtained from the three-term recurrence relations (6) and (8).

\section{The asymmetric tpQRM}

As outlined in Appendix B 1 for the asymmetric onephoton QRM, we can also find the nondegenerate exceptional solutions in the spectra for the asymmetric tpQRM by the pole structures of the $G$ function. When the energy levels cross the pole lines, the coefficients in the $G$ function would diverge and, therefore, should be treated specially. For any real physical systems, the wave function should be analytic, so the numerators $f_{m}^{(q)}$ in Eq. (26) or $c_{m}^{(q)}$ in Eq. (29) should also vanish, which further gives the condition for the model parameters $g, \Delta, \epsilon$ for the fixed value of $m$ associated with one pole line.

In parallel to the asymmetric one-photon QRM, the first nondegenerate exceptional $G$ function associated with the $N$ th type- $A$ pole lines (33) for the asymmetric tpQRM is easily given by

$$
\begin{aligned}
G_{\mathrm{non}, 1 A}^{(q)}= & {\left[\sum_{n=0}^{N-1} \frac{\Delta f_{n}^{(q)} \Omega_{n}^{(q)} / 2}{2 \beta(n-N)}+e_{N} \Omega_{N}^{(q)}\right] } \\
& \times\left[\sum_{n=0}^{\infty} \frac{\Delta c_{n}^{(q)} \Omega_{n}^{(q)} / 2}{2 \beta(n-N)-\epsilon}\right] \\
& -\sum_{n=0}^{N-1} f_{n}^{(q)} \Omega_{n}^{(q)} \sum_{n=0}^{\infty} c_{n}^{(q)} \Omega_{n}^{(q)} \\
= & 0,
\end{aligned}
$$

where

$$
e_{N}^{(q)}=-\frac{4 g \omega}{\Delta \beta} f_{N-1}^{(q)},
$$

and that associated with the $M$ th type- $B$ pole lines (34) reads

$$
\begin{aligned}
G_{\mathrm{non}, 1 B}^{(q)}= & {\left[\sum_{n=0}^{\infty} \frac{\Delta f_{n}^{(q)} \Omega_{n}^{(q)} / 2}{2 \beta(n-M)+\epsilon}\right] } \\
& \times\left[\sum_{n=0}^{M-1} \frac{\Delta c_{n}^{(q)} \Omega_{n}^{(q)} / 2}{2 \beta(n-M)}+d_{M} \Omega_{M}^{(q)}\right] \\
& -\sum_{n=0}^{\infty} f_{n}^{(q)} \Omega_{n}^{(q)} \sum_{n=0}^{M-1} c_{n}^{(q)} \Omega_{n}^{(q)} \\
= & 0,
\end{aligned}
$$


where

$$
d_{M}^{(q)}=-\frac{4 g \omega}{\Delta \beta} c_{M-1}^{(q)} .
$$

Note that by Eqs. (B5) and (B7), all the remaining coefficients for $n>N[n>M]$ vanish. Zeros of the first nondegenerate exceptional $G$ functions are equivalent to $f_{N}^{(q)}=0$ or $c_{M}^{(q)}=0$ . Obviously, the later ones are obviously simpler in practical calculations, whereas the former ones are more conceptually interesting, both can give the same solutions.

Similarly, the second nondegenerate exceptional $G$ function associated with the type- $A$ pole lines (33) is

$$
\begin{aligned}
G_{\mathrm{non}, 2 A}^{(q)}= & {\left[\Omega_{N}^{(q)}+\sum_{n=N+1}^{\infty} \frac{\Delta f_{n}^{(q)} \Omega_{n}^{(q)} / 2}{2 \beta(n-N)}\right] } \\
& \times\left[\sum_{n=0}^{\infty} \frac{\Delta c_{n}^{(q)} \Omega_{n}^{(q)} / 2}{2 \beta(n-N)-\epsilon}\right] \\
& -\sum_{n=N+1}^{\infty} f_{n}^{(q)} \Omega_{n}^{(q)} \sum_{n=0}^{\infty} c_{n}^{(q)} \Omega_{n}^{(q)} \\
= & 0,
\end{aligned}
$$

where we have set $e_{N}^{(q)}=1$ and the coefficients $e_{n<N}^{(q)}=0$ and $f_{n \leqslant N}^{(q)}=0$. By the recurrence relations and the pole energy, all other coefficients can be obtained. The second nondegenerate exceptional $G$ function associated with the type- $B$ pole lines (34) can be obtained in a straightforward way as

$$
\begin{aligned}
G_{\mathrm{non}, 2 B}^{(q)}= & {\left[\sum_{n=0}^{\infty} \frac{\Delta f_{n}^{(q)} \Omega_{n}^{(q)} / 2}{2 \beta(n-M)+\epsilon}\right] } \\
& \times\left[\Omega_{M}^{(q)}+\sum_{n=M+1}^{\infty} \frac{\Delta c_{n}^{(q)} \Omega_{n}^{(q)} / 2}{2 \beta(n-M)}\right] \\
& -\sum_{n=0}^{\infty} f_{n}^{(q)} \Omega_{n}^{(q)} \sum_{n=M+1}^{\infty} c_{n}^{(q)} \Omega_{n}^{(q)} \\
= & 0,
\end{aligned}
$$

where $d_{M}^{(q)}=1$ and the coefficients $d_{n<M}^{(q)}=0$ and $c_{n \leqslant M}^{(q)}=0$.
[1] I. I. Rabi, Space quantization in a gyrating magnetic field, Phys. Rev. 51, 652 (1937).

[2] D. Braak, Q.-H. Chen, M. Batchelor, and E. Solano, Semiclassical and quantum Rabi models: in celebration of 80 years, J. Phys. A: Math. Theor. 49, 300301 (2016).

[3] M. O. Scully and M. S. Zubairy, Quantum Optics (Cambridge University Press, Cambridge, UK, 1997); M. Orszag, Quantum Optics Including Noise Reduction, Trapped Ions, Quantum Trajectories, and Decoherence, 2nd ed. (Springer, Berlin, 2007).

[4] T. Niemczyk, F. Deppe, H. Huebl et al., Circuit quantum electrodynamics in the ultrastrong-coupling regime, Nat. Phys. 6, 772 (2010)

[5] P. Forn-Díaz, J. J. García-Ripoll, B. Peropadre, J.-L. Orgiazzi, M. A. Yurtalan, R. Belyansky, C. M. Wilson, and A. Lupascu, Ultrastrong coupling of a single artificial atom to an electromagnetic continuum in the nonperturbative regime, Nat. Phys. 13, 39 (2017).

[6] D. Leibfried, R. Blatt, C. Monroe, and D. Wineland, Quantum dynamics of single trapped ions, Rev. Mod. Phys. 75, 281 (2003).

[7] K. Hennessy et al., Quantum nature of a strongly coupled single quantum dot-cavity system, Nature (London) 445, 896 (2007).

[8] P. Forn-Díaz, J. Lisenfeld, D. Marcos, J. J. García-Ripoll, E. Solano, C. J. P. M. Harmans, and J. E. Mooij, Observation of the Bloch-Siegert Shift in a Qubit-Oscillator System in the Ultrastrong Coupling Regime, Phys. Rev. Lett. 105, 237001 (2010).

[9] F. Yoshihara, T. Fuse, S. Ashhab, K. Kakuyanagi, S. Saito, and K. Semba, Superconducting qubit-oscillator circuit beyond the ultrastrong-coupling regime, Nat. Phys. 13, 44 (2017).

[10] P. Forn-Díaz, L. Lamata, E. Rico, J. Kono, and E. Solano, Ultrastrong coupling regimes of light-matter interaction, Rev. Mod. Phys. 91, 025005 (2019).
[11] Z. Chen, Y. M. Wang, T. F. Li, L. Tian, Y. Qiu, K. Inomata, F. Yoshihara, S. Han, F. Nori, J. S. Tsai, and J. Q. You, Single-photon-driven high-order sideband transitions in an ultrastrongly coupled circuit-quantum-electrodynamics system, Phys. Rev. A 96, 012325 (2017).

[12] P. Bertet, I. Chiorescu, G. Burkard, K. Semba, C. J. P. M. Harmans, D. P. DiVincenzo, and J. E. Mooij, Dephasing of a Superconducting Qubit Induced by Photon Noise, Phys. Rev. Lett. 95, 257002 (2005)

[13] S. Felicetti, D. Z. Rossatto, E. Rico, E. Solano, and P. FornDíaz, Two-photon quantum Rabi model with superconducting circuits, Phys. Rev. A 97, 013851 (2018).

[14] L. W. Duan, Y.-F. Xie, D. Braak, and Q.-H. Chen, Two-photon Rabi model: analytic solutions and spectral collapse, J. Phys. A: Math. Theor. 49, 464002 (2016).

[15] S. Felicetti, J. S. Pedernales, I. L. Egusquiza, G. Romero, L. Lamata, D. Braak, and E. Solano, Spectral collapse via two-phonon interactions in trapped ions, Phys. Rev. A 92, 033817 (2015).

[16] D. Braak, Integrability of the Rabi Model, Phys. Rev. Lett. 107, 100401 (2011).

[17] Q. H. Chen, C. Wang, S. He, T. Liu, and K. L. Wang, Exact solvability of the quantum Rabi model using Bogoliubov operators, Phys. Rev. A 86, 023822 (2012).

[18] H.-H. Zhong, Q.-T. Xie, M. Batchelor, and C.-H. Lee, Analytical eigenstates for the quantum Rabi model, J. Phys. A: Math. Theor. 46, 415302 (2013); H.-H. Zhong, Q.-T. Xie, X. Guan, M. T. Batchelor, K. Gao, and C.-H. Lee, Analytical energy spectrum for hybrid mechanical systems, ibid. 47, 045301 (2014).

[19] A. J. Maciejewski, M. Przybylska, and T. Stachowiak, Full spectrum of the Rabi model, Phys. Lett. A 378, 16 (2014).

[20] Y. Y. Zhang, Q. H. Chen, and Y. Zhao, Generalized rotating-wave approximation to biased qubit-oscillator systems, Phys. Rev. A 87, 033827 (2013). 
[21] H. Wang, S. He, L. W. Duan, and Q. H. Chen, Solutions to the quantum Rabi model with two equivalent qubits, Europhys. Lett. 106, 54001 (2014).

[22] Z.-J. Ying, M. X. Liu, H.-G. Luo, H.-Q. Lin, and J. Q. You, Ground-state phase diagram of the quantum Rabi model, Phys. Rev. A 92, 053823 (2015).

[23] L. W. Duan, S. He, D. Braak, and Q. H. Chen, Solution of the two-mode quantum Rabi model using extended squeezed states, Europhys. Lett. 112, 34003 (2015).

[24] Z. M. Li and M. T. Batchelor, Algebraic equations for the exceptional eigenspectrum of the generalized Rabi model, J. Phys. A: Math. Theor. 48, 454005 (2015); Addendum to 'Algebraic equations for the exceptional eigenspectrum of the generalized Rabi model', ibid. 49, 369401 (2016).

[25] Z. G. Lv, C. J. Zhao, and H. Zheng, Quantum dynamics of two-photon quantum Rabi model, J. Phys. A: Math. Theor. 50, 074002 (2017).

[26] L. Cong, X. M. Sun, M. X. Liu, Z. J. Ying, and H. G. Luo, Polaron picture of the two-photon quantum Rabi model, Phys. Rev. A 99, 013815 (2019).

[27] Y. F. Xie, X. Y. Chen, X. F. Dong, and Q. H. Chen, First-order and continuous quantum phase transitions in the anisotropic quantum Rabi-Stark model, Phys. Rev. A 101, 053803 (2020); X. Y. Chen, Y. F. Xie, and Q. H. Chen, Quantum criticality of the Rabi-Stark model at finite frequency ratios, ibid. 102, 063721 (2020).

[28] Q.-T. Xie, H.-H. Zhong, M. T. Batchelor, and C.-H. Lee, The quantum Rabi model: solution and dynamics, J. Phys. A: Math. Theor. 50, 113001 (2017).

[29] A. Le Boité, Theoretical methods for ultrastrong lightmatter interactions, Adv. Quantum Technol. 3, 1900140 (2020).

[30] M.-S. Choi, Quantum states of circuit quantum electrodynamics in the ultra-strong coupling regime, Adv. Quantum Technol. 3, 2000085 (2020).

[31] B. R. Judd, Exact solutions to a class of Jahn-Teller systems, J. Phys. C: Solid State Phys. 12, 1685 (1979).

[32] C. Emary and R. F. Bishop, Bogoliubov transformations and exact isolated solutions for simple nonadiabatic Hamiltonians, J. Math. Phys. 43, 3916 (2002); Exact isolated solutions for the two-photon Rabi Hamiltonian, J. Phys. A 35, 8231 (2002).

[33] M. Wakayama, Symmetry of asymmetric quantum Rabi models, J. Phys. A: Math. Theor. 50, 174001 (2017); K. Kimoto, C. Reyes-Bustos, and M. Wakayama, Determinant expressions of constraint polynomials and the spectrum of the asymmetric quantum Rabi model, Int. Math. Res. Not. 2021, 9458 (2021).

[34] S. Ashhab, Attempt to find the hidden symmetry in the asymmetric quantum Rabi model, Phys. Rev. A 101, 023808 (2020).

[35] V. V. Mangazeev, M. T. Batchelor, and V. V. Bazhanov, The hidden symmetry of the asymmetric quantum Rabi model, J. Phys. A: Math. Theor. 54, 12LT01 (2021).

[36] C. Reyes-Bustos, D. Braak, and M. Wakayama, Remarks on the hidden symmetry of the asymmetric quantum Rabi model, J. Phys. A: Math. Theor. 54, 285202 (2021).

[37] A. J. Maciejewski and T. Stachowiak, Level crossings and new exact solutions of the two-photon Rabi model, J. Phys. A: Math. Theor. 52, 485303 (2019).

[38] Q. T. Xie, Exact solution of the two-mode quantum Rabi model, Commun. Theor. Phys. 72, 065105 (2020).

[39] Z. M. Li and M. T. Batchelor, Hidden symmetry and tunneling dynamics in asymmetric quantum Rabi models, Phys. Rev. A 103, 023719 (2021).

[40] Q. H. Chen, Y. Y. Zhang, T. Liu, and K. L. Wang, Numerically exact solution to the finite-size Dicke model, Phys. Rev. A 78, 051801(R) (2008).

[41] D. Braak, Symmetries in the quantum Rabi model, Symmetry 11, 1259 (2019).

[42] I. Travěnec, Solvability of the two-photon Rabi Hamiltonian, Phys. Rev. A 85, 043805 (2012); A. J. Maciejewski, M. Przybylska, and T. Stachowiak, Comment on Solvability of the two-photon Rabi Hamiltonian', ibid. 91, 037801 (2015); I. Travěnec, Reply to "Comment on 'Solvability of the twophoton Rabi Hamiltonian”, ibid. 91, 037802 (2015).

[43] S. Cui, J. P. Cao, H. Fan, and L. J. Amico, Exact analysis of the spectral properties of the anisotropic two-bosons Rabi model, J. Phys. A: Math. Theor. 50, 204001 (2017).

[44] M. T. Batchelor, Z. M. Li, and H. Q. Zhong, Energy landscape and conical intersection points of the driven Rabi model, J. Phys. A: Math. Theor. 49, 01LT01 (2016).

[45] Z. M. Li, D. Ferri, and M. T. Batchelor, Nonorthogonal-qubitstate expansion for the asymmetric quantum Rabi model, Phys. Rev. A 103, 013711 (2021).

[46] X. Y. Chen, L. W. Duan, D. Braak, and Q. H. Chen, Multiple ground-state instabilities in the anisotropic quantum Rabi model, Phys. Rev. A 103, 043708 (2021).

[47] D. Braak in Proceedings of the Forum of Mathematics for Industry 2014, edited by R. S. Anderssen et al. (Springer, Japan, 2016), p. 75. 